\title{
MAXIMAL ABELIAN SUBALGEBRAS WITH SIMPLE NORMALIZER
}

\author{
ROBERTO LONGO
}

(Communicated by John B. Conway)

\begin{abstract}
All infinite factors with separable predual contain a maximal Abelian
* subalgebra whose normalizer generates a simple subfactor
\end{abstract}

\section{INTRODUCTION}

The purpose of this note is to point out that every infinite factor $M$, with separable predual, contains a maximal Abelian ${ }^{*}$ subalgebra $A$ whose normalizer $\mathscr{N}(A)$ generates a simple subfactor of $M$.

We recall that a subfactor $N \subset M$ is simple in $M$ if $N \vee J N J=B\left(L^{2}(M)\right)$ where $J$ is the modular conjugation of $L^{2}(M)$ (the lattice symbol $\vee$ denotes the von Neumann algebra generated). We refer to $[2,3]$ for the properties of simple subfactors; what we need to know here is that $M$ always contains a simple injective subfactor.

The proof of our result closely follows an argument of Popa [4, p. 160] with one crucial difference: we use simple injective subfactors at the place of injective subfactors with trivial relative commutant [1].

In this way we obtain a superposition of the results in $[2,4]$ providing the general construction of a new kind of MASA whose properties are more stringent than those shared by semiregular MASA's [4]. ( $A$ is semiregular if $\mathscr{N}(A)^{\prime \prime}$ is a factor; this factor has automatically trivial relative commutant in $M$ since it contains $A$. One calls $A$ regular if $\mathscr{N}(A)^{\prime \prime}=M$.)

\section{THE CONSTRUCTION}

Let $\mathscr{H}$ be a separable Hilbert space. We choose a bounded metric $d$ on the unit ball $B(\mathscr{H})_{1}$ on $B(\mathscr{H})$, inducing the strong topology, and a strongly dense sequence $\left\{x_{n}\right\}$ in $B(\mathscr{C})_{1}$. If $N \subset B(\mathscr{C})$ is a von Neumann algebra we put

$$
\delta(N) \equiv \sum_{i=1}^{\infty} \frac{d\left(x_{i}, N\right)}{2^{i}}
$$

Received by the editors July 26, 1988 and, in revised form October 28, 1988.

1980 Mathematics Subject Classification (1985 Revision). Primary 46L10; Secondary 46L35.

Key words and phrases. von Neumann algebras, subfactors, MASA.

Supported in part by Ministero della Pubblica Istruzione and CNR-GNAFA. 
where $d(\cdot, N)$ denotes the distance from the unit ball of $N$.

If $N_{k}$ is an increasing sequence of von Neumann algebras then $\delta\left(N_{k}\right) \rightarrow 0$ iff $\vee N_{k}=B(\mathscr{H})$.

Let now $M$ be an infinite factor on $\mathscr{H}$ with a cyclic separating vector $\Omega$ and modular conjugation $J$. If $A \subset M$ is an Abelian von Neumann subalgebra we put

$$
\eta(A)=d\left([A \Omega], A \vee M^{\prime}\right),
$$

where $e=[A \Omega] \in A^{\prime}$ denotes the projection onto the closure of $A \Omega$.

The following lemma is contained in $[4,5]$ and is included for convenience; the other lemmas are standard or elementary.

Lemma 1. Let $A_{k}$ be an increasing sequence of Abelian von Neumann subalgebras with $A=\vee A_{k}$.

(i) $A$ is maximal Abelian in $M$ iff $\eta(A)=0$ i.e. $e \in A \vee M^{\prime}$;

(ii) $\eta\left(A_{k}\right) \rightarrow \eta(A)$.

Hence $A$ is a MASA of $M$ iff $\eta\left(A_{k}\right) \rightarrow 0$.

Proof.

(i) If $A$ is a MASA of $M$, then $A^{\prime} \cap M=A$ namely $A^{\prime}=A \vee M^{\prime}$ and $e \in A \vee M^{\prime}$. Conversely assume $e \in A \vee M^{\prime}$ and notice that the reduced von Neumann algebra $A_{e}$ is maximal Abelian in $B(e \mathscr{H})$, i.e. $A_{e}^{\prime}=A_{e}$, due to the cyclicity of $\Omega$ for $A_{e}$. Since $A^{\prime} \cap M \supset A$ or $A^{\prime} \supset A \vee M^{\prime}$ we have

$$
A_{e}=A_{e}^{\prime} \supset\left(A \vee M^{\prime}\right)_{e} \supset A_{e}
$$

thus $\left(A \vee M^{\prime}\right)_{e}=A_{e}$ or $\left(A^{\prime} \cap M\right)_{e}=A_{e}$ that implies $A^{\prime} \cap M=A$ because $\Omega$ is separating for $M$.

(ii) The sequence of projections $e_{k}=\left[A_{k} \Omega\right]$ converges to $e$ increasingly, hence strongly, and $d\left(e_{k}, e\right) \rightarrow 0$; we have

$$
\begin{gathered}
\eta(A)-\eta\left(A_{k}\right)=d\left(e, A \vee M^{\prime}\right)-d\left(e_{k}, A_{k} \vee M^{\prime}\right) \\
=\left[d\left(e, A \vee M^{\prime}\right)-d\left(e, A_{k} \vee M^{\prime}\right)\right]+\left[d\left(e, A_{k} \vee M^{\prime}\right)-d\left(e_{k}, A_{k} \vee M^{\prime}\right)\right] \\
\leq d\left(e, A \vee M^{\prime}\right)-d\left(e, A_{k} \vee M^{\prime}\right)+d\left(e, e_{k}\right) \rightarrow 0
\end{gathered}
$$

where $d\left(e, A_{k} \vee M^{\prime}\right) \rightarrow d\left(e, A \vee M^{\prime}\right)$ because $A_{k} \vee M^{\prime}$ increases to $A \vee M^{\prime}$.

Lemma 2. Let $N$ be an infinite factor with separable predual. There exists an increasing sequence of discrete Abelian von Neumann algebras $B_{n}$ such that $B=\vee B_{n}$ is maximal Abelian in $N$ and all the atoms of $B_{n}$ are infinite (thus equivalent) projections of $N$.

Proof. The statement is clear in the case of a type I factor $F$ (consider the step function approximation of $L^{\infty}[0,1]$ and regard it as a MASA of $B\left(L^{2}[0,1]\right)$ as usual). For a general infinite factor $N$ note that for any MASA $B$ of $N$ the isomorphism of the diffuse part of $B$ (if nonzero) with $L^{\infty}[0,1]$ makes possible an atomic approximation that we only need to adjust in order that 
all projections be infinite. Since $N$ is isomorphic to $N \otimes F$ we may achieve this by tensoring $B$ with a MASA of $F$ as above (the tensor product of two projections is an infinite projection if one of them is infinite).

Lemma 3. Let $N$ be a factor and $B$ a discrete Abelian von Neumann subalgebra of $N$. If all the atoms of $B$ are equivalent, there exists a type $I$ subfactor $G$ of $N$ such that $B$ is a MASA of $G$. If the atoms of $B$ are infinite projections, then $G^{\prime} \cap N$ is an infinite factor.

Proof. Let $\left\{p_{i}, i \in I\right\}$ be the atoms of $B$; fix $i_{0} \in I$ and choose a partial isometry $v_{i} \in N$ from $p_{i_{0}}$ to $p_{i}, i \in I$. Then $\left\{v_{i} v_{j}^{*} ; i, j \in I\right\}$ is a system of matrix units for $G$. As usual $N$ is isomorphic to $N_{p_{i_{0}}} \otimes G$ hence $G^{\prime} \cap N$ is isomorphic to $N_{p_{i_{0}}}$ which is an infinite factor iff $p_{i_{0}}$ is an infinite projection.

Lemma 4. Let $N_{i}$ be a subfactor of the factor $M_{i}(i=1,2)$. The subfactor $N_{1} \otimes N_{2}$ of $M_{1} \otimes M_{2}$ is simple iff $N_{1}$ is simple in $M_{1}$ and $N_{2}$ is simple in $M_{2}$. Proof. Let $J_{i}$ be the modular conjugation of $L^{2}\left(M_{i}\right)$, so that $J=J_{1} \otimes J_{2}$ is the modular conjugation of $L^{2}\left(M_{1} \otimes M_{2}\right)=L^{2}\left(M_{1}\right) \otimes L^{2}\left(M_{2}\right)$. We have

$$
\left(N_{1} \otimes N_{2}\right) \vee J\left(N_{1} \otimes N_{2}\right) J=\left(N_{1} \vee J_{1} N_{1} J_{1}\right) \otimes\left(N_{2} \vee J_{2} N_{2} J_{2}\right)
$$

that readily entails the statement.

Theorem 5. Let $M$ be an infinite factor with separable predual. There exists a maximal Abelian " subalgebra $A$ of $M$ whose normalizer $\mathcal{N}(A)$ generates a simple subfactor $\mathscr{N}(A)^{\prime \prime}$ of $M$.

Proof. We order the pairs $(A, F)$ consisting of a type $I$ sufactor $F$ of $M$ with infinite relative commutant $F^{\prime} \cap M$ and a maximal Abelian ${ }^{*}$ subalgebra $A$ of $F$ in such a way that $(A, F) \subset(\tilde{A}, \tilde{F})$ means that $F \subset \tilde{F}$ and $\tilde{A}=A \vee B$ with $B$ a MASA of $F^{\prime} \cap \tilde{F}$ (in other words $(A, F)$ is a tensor product component of $(\tilde{A}, \tilde{F}))$. We will construct an increasing sequence of pairs $\left(A_{k}, F_{k}\right)$ with

$$
\eta\left(A_{k}\right) \rightarrow 0, \quad \delta\left(F_{k} \vee J F_{k} J\right) \rightarrow 0
$$

that will prove the theorem because $A=\vee A_{k}$ will be a MASA of $M$ by Lemma 1 and $\mathscr{N}(A)^{\prime \prime} \supset R$ where $R=\vee F_{k}$ is simple injective subfactor of $M$.

By an iterative argument it suffices to prove separately that, for any given pair $(A, F)$, there exists a pair $(\tilde{A}, \tilde{F}) \supset(A, F)$ such that

(a) $\eta(\tilde{A}) \leq \frac{1}{2} \eta(A)$

(b) $\delta(\tilde{F} \vee J \tilde{F} J) \leq \frac{1}{2} \delta(F \vee J F J)$.

To prove a) we choose in the factor $F^{\prime} \cap M$ an increasing sequence of discrete Abelian von Neumann subalgebras $B_{n}$ such that $\vee B_{n}$ is maximal Abelian and all the atoms of $B_{n}$ are infinite, thus equivalent, in $F^{\prime} \cap M$ (Lemma 2).

Since $A \vee B_{n}$ increases to a MASA of $M$ we have $\eta\left(A \vee B_{n}\right) \rightarrow 0$. Let $m$ be so large that $\eta\left(A \vee B_{m}\right) \leq \frac{1}{2} \eta(A)$ and let $G$ be a type $I$ subfactor of $F^{\prime} \cap M$ containing $B_{m}$ as a MASA and notice that the relative commutant of $G$ in 
$F^{\prime} \cap M$ is infinite (Lemma 3). The pair $(\tilde{A}, \tilde{F})$ with $\tilde{A}=A \vee B_{m}, \tilde{F}=F \vee G$ satisfies a).

To prove b) let $R$ be a simple injective subfactor of $F^{\prime} \cap M$ [2]. Because of the tensor product decomposition $M \simeq F \otimes\left(F^{\prime} \cap M\right)$ the subfactor $F \vee R$ of $M$ is simple and injective (Lemma 4).

Let $\left\{F_{n}\right\}$ be an increasing sequence of type $I$ subfactors of $M$, with $F=F_{1}$ and $F_{n}^{\prime} \cap M$ infinite, that generates $R$. Since $\delta\left(F_{n} \vee J F_{n} J\right) \rightarrow 0$ we may chose $m$ so large that $\delta\left(F_{m} \vee J F_{m} J\right) \leq \frac{1}{2} \delta(F \vee J F J)$. Choose a MASA $B$ of $F^{\prime} \cap F_{m}$ and set $\tilde{A}=A \vee B, \tilde{F}=F_{m}$ so that $(\tilde{A}, \tilde{F}) \supset(A, F)$ and step $\left.\mathrm{b}\right)$ is done.

Remarks. Let $A$ be MASA of $M$ as in the theorem:

(a) If there exists a normal conditional expectation on $\varepsilon$ of $M$ onto $A$ then $A$ is a Cartan subalgebra of $M$. In fact if $\phi$ is a faithful normal state such that its modular group $\sigma^{\phi}$ leaves $A$ invariant, then $\sigma^{\phi}$ leaves $\mathcal{N}(A)^{\prime \prime}$ invariant, therefore by Takesaki criterium there exists a normal conditional expectation of $M$ onto $\mathscr{N}(A)^{\prime \prime}$ which implies $\mathscr{N}(A)^{\prime \prime}=M$ [2].

(b) Since $\mathscr{N}(A)$ determines the automorphisms of $M$ [2], it is possible to analyze the automorphism group of the pair as in [6]. For example denote by $\operatorname{Aut}(M \mid A) / \operatorname{Inn}(M \mid A)$ the group of automorphisms of $M$ leaving $A$ pointwise invariant modulo the corresponding inner automorphism subgroup; given $\alpha \in$ $\operatorname{Aut}(M \mid A)$ the map $u \in \mathscr{N}(A) \rightarrow Z_{u}^{\alpha} \equiv \alpha(u) u^{*}$ is an ad-cocycle with values in $A$, that induces an isomorphism of $\operatorname{Aut}(M \mid A) / \operatorname{Inn}(M \mid A)$ into cohomology group $H_{\text {ad }}^{1}(\mathscr{N}(A), A)$.

(c) The proof of the theorem shows that there exists a simple injective subfactor $R \subset M$ such that $A$ is a regular MASA of $R$. If $M$ is already approximately finite-dimensional one obtains (by a slight variation of the argument) the known result that $M$ contains a regular MASA.

\section{REFERENCES}

1. R. Longo, Solution of the factorial Stone-Weierstrass conjecture. An application of the theory of standard split inclusions, Invent. Math. 76 (1984), 145-155.

2. __ Simple injective subfactors, Adv. in Math. 63 (1987), 152-171.

3. __ Restricting a compact action to an injective subfactor, Ergodic Theory and Dynamical Systems (to appear).

4. S. Popa, Semiregular maximal abelian subalgebras and the solution of the factor state StoneWeierstrass problem, Invent. Math. 76 (1984), 157-161.

5. C. Skau, Finite subalgebras of a von Neumann algebra, J. Funct. Anal. 25 (1977), 211-235.

6. I. M. Singer, Automorphisms of finite factors, Amer. J. Math. 77 (1955), 117-133.

Dipartimento di Matematica, Universitá di Roma II "Tor Vergata," Roma, Italy 